\title{
Sonic Hedgehog/Patch Pathway Signaling Status Unknown
}

National Cancer Institute

\section{Source}

National Cancer Institute. Sonic Hedgehog/Patch Pathway Signaling Status Unknown. NCI Thesaurus. Code C82327.

The result of the sonic hedgehog/patch pathway signaling status is not known or the test has not been performed. 